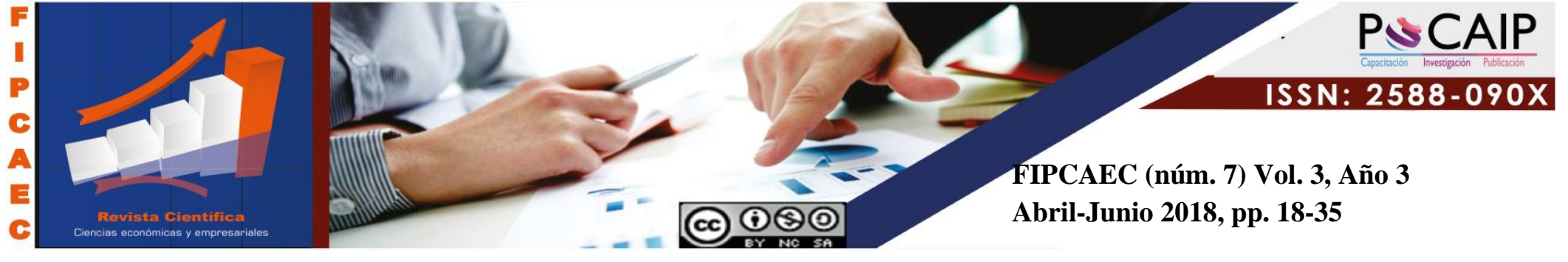

DOI: DOI 10.23857/fipcaec.v3i7.90

\title{
Análisis estratégico en pequeñas empresas
}

Strategic analysis in small businesses

Análise estratégica em pequenas empresas

\author{
Emilio Macías Guadamud ${ }^{1}$ \\ emiliomaciasguadamud@gmail.com \\ https://orcid.org/0000-0001-6659-0494
}

Correspondencia: emiliomaciasguadamud@gmail.com

* Recepción: 10/ 01/2018 *Aceptación: 12/03/ 2018 *Publicación: 15 /04/ 2018

1 Especialista en Diseño Curricular por Competencia, Ingeniero Comercial, Docente de la Universidad Laica Eloy Alfaro de Manabí, Facultad de Ciencias Administrativas, Manta, Ecuador. 


\title{
Resumen
}

La investigación tuvo por objetivo analizar la factibilidad de un plan estratégico en pequeñas empresas, específicamente en automotriz Gribel, siendo de tipo descriptiva con una población maestral de 15 personas que laboran en la automotriz Gribel, ubicada en la ciudad de Manta Ecuador. A pesar de que la empresa tiene objetivos claros y conocidos por la mayoría del personal, la mayoría de la muestra no participo en su creación. Estos resultados confirman la disposición de cambio y transformación evidenciados en la muestra estudiada, dado el alto porcentaje de la misma que insiste en la creación de nuevos procedimientos que sean conocidos y manejados por todos. Se concluye que es necesario el diseño de un plan estratégico para la empresa objeto de estudio, por cuanto los principios de la planificación estratégica le permitirán a los trabajadores estar capacitados para resolver cualquier eventualidad y potenciar así el crecimiento de la empresa.

Palabras clave: Planificación de programas; diseño de proyecto; estudio de viabilidad; proyecto piloto.

\begin{abstract}
The objective of the research was to analyze the feasibility of a strategic plan in small businesses, specifically in the Gribel automotive, being descriptive type with a master population of 15 people working in the Gribel automotive, located in the city of Manta - Ecuador. Although the company has clear objectives and known by the majority of the staff, the majority of the sample did not participate in its creation. These results confirm the change and transformation disposition evidenced in the sample studied, given the high percentage of it that insists on the creation of new procedures that are known and managed by all. It is concluded that the design of a strategic plan for the company under study is necessary, since the principles of strategic planning will allow workers to be trained to resolve any eventuality and thus enhance the growth of the company.
\end{abstract}

Keywords: Program planning; project design; viability study; pilot project. 


\section{Resumo}

O objetivo da pesquisa foi analisar a viabilidade de um plano estratégico em pequenas empresas, especificamente no setor automotivo Gribel, sendo do tipo descritivo com uma população mestra de 15 pessoas que trabalham no setor automotivo Gribel, localizado na cidade de Manta Equador. Embora a empresa tenha objetivos claros e conhecidos pela maioria dos funcionários, a maioria da amostra não participou de sua criação. Esses resultados confirmam a disposição de mudança e transformação evidenciada na amostra estudada, dada a alta porcentagem que insiste na criação de novos procedimentos que são conhecidos e gerenciados por todos. Conclui-se que é necessário o desenho de um plano estratégico para a empresa em estudo, uma vez que os princípios do planejamento estratégico permitirão que os trabalhadores sejam treinados para resolver qualquer eventualidade e, assim, aumentar o crescimento da empresa.

Palavras-chave: Planejamento de programas; concepção do projeto; estudo de viabilidade; projeto piloto.

\section{Introducción}

Con el fin de realizar cualquier tipo de actividad empresarial hoy en día es necesario que los gerentes de empresa tengan un mínimo de conocimiento acerca de competitividad, y de las normas y procedimientos para poder llevar a cabo de manera formal la gerencia de una organización. Los gerentes se encuentran permanentemente en búsqueda de incrementar la competitividad y para adaptarse a los cambios en el entorno es frecuente el hecho de que las compañías se reestructuren, cambien los métodos de producción y procuren incrementar su capacidad productiva de acuerdo a sus posibilidades económicas.

Las organizaciones actualmente enfrentan cambios constantes dentro de su entorno a los cuales tiene que adaptarse para poder sobrevivir, esto se debe a la globalización de los mercados, el desarrollo de la tecnología que a su vez impulsan a las organizaciones a identificar y desarrollar ventajas competitivas, ya que de esa manera podrán brindar servicios o productos de calidad. Es válido decir entonces que estas empresas asumen el reto de establecer o crear estrategias donde la eficacia y la eficiencia son requerimientos indispensables para poder elevar la calidad de los 
bienes y servicios que prestan y de esta manera posicionarse en el mercado en el cual llevan adelante sus operaciones comerciales.

Al respecto de lo anterior Kotler \& Lee (2011), afirma que la planeación estratégica es el proceso gerencial de desarrollar y mantener una dirección que pueda alinear las metas y recursos de la organización con sus oportunidades cambiantes de mercadeo. Por lo tanto, la planeación estratégica es necesaria para que las organizaciones logren ventajas competitivas que les permitan prevalecer y permanecer a lo largo del tiempo.

Ante la necesidad de realizar un análisis de la situación inicial, se debe evaluar el entorno empresarial, de acuerdo a Kotler \& Lee (2011), es el estudio de las circunstancias que afectan a la empresa, las cuales al variar la afectan por lo que se hace necesario para la organización el ser flexible ante los cambios en los aspectos políticos, económicos, legales, tecnológicos y sociales. Por lo tanto el análisis del entorno empresarial viene a ser necesario para las organizaciones porque le permite plantearse objetivos a corto y mediano plazo que pueden influir en el entorno o al menos minimizar las consecuencias negativas de un cambio en el mismo.

Es así que la investigación tiene por objetivo analizar la factibilidad de un plan estratégico en pequeñas empresas, específicamente en automotriz Gribel, ubicada en la ciudad de Manta Ecuador, con la finalidad de proveer de estrategias para la consolidación de la organización.

\section{Desarrollo}

\section{Plan estratégico}

De acuerdo a Fred (2013), "el plan estratégico se define como el arte y la ciencia de formular, implementar, y evaluar decisiones multifuncionales que le permitan a una organización lograr sus objetivos", también detalla la finalidad con estas palabras: "La finalidad de la planeación estratégica es aprovechar las oportunidades existentes y crear otras nuevas y diferentes para el futuro; en contraste la planeación a largo plazo busca optimizar para el mañana las tendencias de hoy. Por otra parte el autor Sainz De Vicuña (2012), al abordar el plan estratégico de una organización, se refiere al plan maestro en el que la alta dirección recoge las decisiones estratégicas corporativas que se han adoptado hoy, en referencia a lo que hará en los tres 
próximos años.

En otras palabras, el planeamiento estratégico es un proceso en la medida que reúne una serie de actividades cuyo fin es la generación de estrategias alternativas hacia el logro de la visión de la organización; además es un instrumento por qué sirve como guía y orientador de la gestión hacia el logro de la visión. Así mismo Martínez y Gutiérrez (2005), mencionan que un plan estratégico es un documento que sintetiza a nivel económico, financiero, estratégico y organizativo el futuro de la empresa, en pocas palabras este se refiere al proceso mediante el cual una organización define su visión de largo plazo y las estrategias para alcanzar a partir del análisis de sus fortalezas, debilidades, oportunidades y amenazas. Supone la participación activa de los autores organizacionales, la obtención permanente de información sobre sus factores claves de éxito, su revisión, monitoreo y ajustes periódicos para que se convierta en un estilo de gestión que haga de la organización un ente proactivo y participativo.

Por otra parte, se resalta que las herramientas fundamentales de planeamiento estratégico, que es el análisis del entorno, es decir, la interacción de la organización con aquello factores que la rodean y determina su comportamiento y además ejercen influencia sobre sus acciones. Esto nos da a entender que a partir de ese análisis se van determinando la visión, misión, objetivos y estrategias de la organización con el fin de orientar sus actividades, adaptarse y anticipar los cambios con mira hacia el futuro.

\section{Importancia del Plan Estratégico}

Es sabido que la perspectiva a largo plazo u horizonte, es la respuesta a la pregunta de ¿En dónde necesita estar la empresa en un momento específico del futuro para desempeñar su visión, misión y estrategias? Para Fred (2013) el Planeamiento Estratégico es un proceso dinámico lo suficientemente flexible para permitir y hasta forzar modificaciones en los planes, a fin de responder a las cambiantes circunstancias. Desde el punto de vista pedagógico el Planeamiento Estratégico es importante porque ofrece a las organizaciones las siguientes ventajas:

- Obliga a los ejecutivos a ver el planeamiento desde la macro perspectiva, señalando los objetivos centrales, de manera que nuestras acciones diarias nos acerquen cada vez más a las metas. 


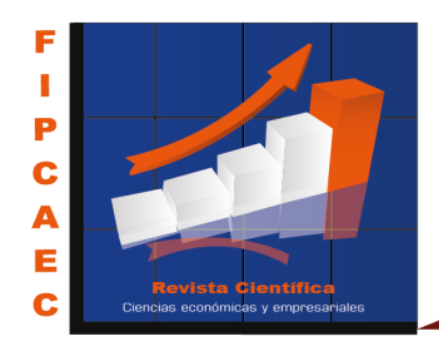

- Mantiene a la vez el enfoque en el futuro y el presente. Refuerza los principios adquiridos en la visión, misión y estrategias.

- Fomenta el planeamiento y la comunicación interdisciplinarios.

- Asigna prioridades en el destino de los recursos.

- Mejora el desempeño de la organización: Orienta de manera efectiva el rumbo de la organización facilitando la acción innovadora de dirección y liderazgo. Permite enfrentar los principales problemas de la organización: Enfrentar el cambio en el entorno y develar las oportunidades y las amenazas.

- El desarrollo de una planificación estratégica produce beneficios relacionados con la capacidad de realizar una gestión más eficiente, liberando recursos humanos y materiales, lo que redunda en la eficiencia productiva y en una mejor calidad de vida y trabajo para todos los miembros. Ayuda a mejorar los niveles de productividad, conducentes al logro de la rentabilidad.

- Favorece a la Dirección y coordinación organizacional al unificar esfuerzos y expectativas colectivas.

- Optimiza los procesos en las Áreas al traducir los resultados en logros sinérgicos.

- Contribuye a la creación y redefinición de valores corporativos.

En función a lo expuesto anteriormente, radica la importancia del plan estratégico para cualquier empresa, como lo es en este caso, dado que persigue como fin último la consolidación de la organización, mediante la evaluación de los logros alcanzados y la reformulación de las políticas de la empresa en caso de que los resultados obtenidos fuesen diferentes de los proyectados.

\section{Características del plan estratégico}

\section{Objetivo de la situación y análisis de los interesados (stakeholder)}

Es necesario mencionar que según Fred (2013), sin una comprensión objetiva e imparcial de "lo que está pasando", no es probable pensar que esta estrategia va a ser muy eficaz. Vale decir que corresponde hacer un análisis que tome en consideración lo que ocurre externa e internamente, y prestar especial atención a las necesidades de los grupos de interés "stakeholder". Por lo tanto es fundamental para la correcta realización de un Plan Estratégico, el análisis detallado de los 
factores internos y externos que inciden positiva o negativamente sobre los intereses de estos grupos de interés, sean estos empleados, miembros de la comunidad, usuarios, proveedores, ente gubernamental o cualquier otro tipo de relación que se pudiere presentar con la organización.

\section{Claridad de objetivos y metas realistas}

Para Ned (2014) es de vital importancia entender el propósito de su iniciativa estratégica y tener metas claras a las que se aspira, pero realistas, señala, el autor y dueño de Foursight Seminars Inc considera que se habla de ello en términos de "propósito, el enfoque y la pasión." Sin embargo la claridad de los propósitos, de la atención y la pasión necesarias para lograr un esfuerzo sostenido y exitoso no es sólo un deseo sino un conjunto de acciones encaminadas a enérgicamente lograr la meta. Es decir que es fundamental para la planificación estratégica que se logre comunicar la información al talento humano con la finalidad de sumar esfuerzos en favor de un objetivo claro y que pueda ser asimilado por todos los involucrados como realizable.

\section{Sentido de urgencia}

En este aspecto Allen (2010), lo describe como "Un nivel a pasar determina tener que actuar hoy, se trata de encender el fuego de la voluntad e inspiración para generar el cambio", así mismo sostiene que: "Sin un sentido de urgencia, es demasiado fácil dejar para mañana lo que se debe hacer hoy“. Esto es relevante, porque el cambio debe hacerse de manera tan expedita como se pueda, entendiendo que este sentido de la urgencia corresponde a la necesidad de solventar cualquier situación adversa o para aprovechar una oportunidad que se presente de manera específica. La finalidad del Plan Estratégico es prever los escenarios y estar preparados para ello.

\section{Estrategias que ponen de relieve sus valores y juegan con sus fortalezas organizativas}

En relación a este aspecto Kenichi (1991), define la estrategia como un plan ideado para brindar a la organización una ventaja competitiva. Por lo que se puede afirmar que la estrategia no se trata de las tareas a realizar, sino de entender lo que haría en base a sus prioridades y valores. Es decir, son aquellas acciones necesarias para brindar una ventaja competitiva, de acuerdo con los valores de la propia organización. Corresponde igualmente señalar que Collins y Porras (1995) hablan de la importancia de equilibrar el núcleo invariable (los valores y el propósito de la empresa) 
mientras estimula el progreso (el cambio y la innovación). Esto permite concluir que la estrategia no solo debe ofrecer una ventaja competitiva, sino que corresponde comprender cuales son los rasgos más importantes dentro de la cultura organizacional específica.

\section{Comprenda su cultura}

Cierto es que la Cultura Organizacional es un elemento condicionante para cualquier Plan Estratégico, Chiavenato (2006) la define como "los valores, las creencias y los principios que constituyen las raíces del sistema gerencial de una organización, así como también al conjunto de procedimientos y conductas gerenciales que sirven de soporte a esos principios básicos”. Es por ello que para lograr el cambio, el plan debe persuadir a todos los involucrados en su ejecución conforme al marco de la organización a la que pertenecen.

\section{Liderazgo}

El liderazgo es el arte de influir en las personas. Es un conjunto de características que el líder persuada a sus seguidores de realizar alguna acción que este considere pertinente. Por lo tanto esta función debe ser ejercida con responsabilidad y es considerada un factor fundamental en el proceso de planificación estratégica.

\section{Disciplina inquebrantable}

Para Rodríguez-Lara (2011) esto se traduce en el logro de los objetivos estratégicos y la ejecución exitosa, lo que permite considerar que significa que existe la disciplina necesaria para alcanzar las metas propuestas y hacer el cambio de comportamiento sostenible. El orden y la disciplina organizacional son también elementos necesarios para la correcta formulación y aplicación de un plan estratégico.

\section{Transparencia}

La gerencia debe proponer el cambio de manera inteligente y honesta con todos los elementos que conforman la organización. 


\section{Seguimiento, medición y retroalimentación}

El seguimiento, medición y control consiste en monitorear el progreso de su plan, recobrar la data de interés y así identificar los elementos que favorecen o amenazan la concreción de un plan. Para los fines de la propuesta correspondiente a la presente investigación la medición de los resultados será parte del diseño de la misma.

\section{Asumir los cambios en la cultura empresarial}

Es de importancia primordial para Chiavenato (2006) definir el cambio organizacional, como el momento en el que el talento humano afronta el mayor reto que es hacerse parte del cambio de manera entusiasta y positiva, transformando la situación problemática en una más favorable. Por ello el Plan Estratégico debe reconocer la relevancia del personal en la obtención de resultados positivos.

\section{Metodología de la investigación}

La investigación desde el aspecto metodológico, siguió los criterios de Hernández, Fernández, Baptista (2014), siendo de tipo descriptiva con diseño no experimental de campo, teniendo una población maestral de 15 personas que laboran en la automotriz Gribel, ubicada en la ciudad de Manta - Ecuador, aplicándose un instrumento tipo dicotómico de 33 ítems de respuestas, aplicándose una prueba piloto obteniéndose el resultado de 0.97 de confiabilidad, mediante el coeficiente KR20 de Kuder-Richardson, recomendándose su debida aplicación, se trabajó con la estadística descriptiva para el análisis de la información. 


\section{Resultados}

\section{Cuadro 1}

Indicador objetivos

ÍTEMS

\begin{tabular}{lllll} 
& $\mathrm{F}$ & $\%$ & $\mathrm{~F}$ & $\%$ \\
\hline $\begin{array}{l}\text { 1. ¿Conoce los objetivos de la } \\
\text { empresa? }\end{array}$ & $73 \%$ & 4 & $27 \%$ \\
$\begin{array}{l}\text { 2. ¿La empresa cuenta con } 11 \\
\text { objetivos claros? }\end{array}$ & $67 \%$ & 5 & $33 \%$ \\
3. ¿Ha participado en la 5 & $67 \%$ & 10 & $33 \%$ \\
creación de los objetivos de & & & \\
la empresa?
\end{tabular}

El $73 \%$ de la empresa conoce los objetivos de la misma mientras que un $27 \%$ no los conoce. Asimismo, se evidencia que para el $67 \%$ de la muestra estos objetivos están claros y para el 33\% no. Sin embargo solo el 33\% de los entrevistados participaron directamente en la creación de los objetivos de la empresa.

Estos resultados muestran que a pesar de que la empresa tiene objetivos claros y conocidos por la mayoría del personal, la mayoría de la muestra no participo en su creación. En este sentido, Koontz \& Weihrich (2011) plantean que los objetivos de un plan estratégico representan los fines hacia los cuales se dirige la actividad que representan no solo la finalidad de la planeación, sino también el fin hacia donde se encamina la organización, su creación debe ser construida por los miembros de la empresa lo que se traducirá a un conocimiento claro de los mismos, promoviendo el sentido de pertenencia en la organización. 


\section{Cuadro 2}

Indicador visión

\begin{tabular}{lllll}
\multicolumn{1}{l}{ ÍTEMS } & Si & \multicolumn{3}{l}{ NO } \\
& & & & \\
& F & $\%$ & F & $\%$ \\
\hline $\begin{array}{l}\text { 4. ¿Considera que existe una visión a futuro } \\
\text { de la empresa? }\end{array}$ & 6 & $40 \%$ & 9 & $60 \%$ \\
$\begin{array}{l}\text { 5. ¿Cree Ud. que el diseño de un plan } \\
\text { estratégico permitirá reforzar esa visión? }\end{array}$ & 13 & $87 \%$ & 2 & $13 \%$ \\
$\begin{array}{l}\text { 6. ¿Cree Ud. que la visión le permitirá } \\
\text { alcanzar el éxito para la empresa? }\end{array}$ & 14 & $93 \%$ & 1 & $7 \%$
\end{tabular}

El $40 \%$ de la muestra afirma que la empresa y su plan tienen una visión establecida mientras que el $60 \%$ afirma no tenerla. Asimismo el $87 \%$ de los participantes afirman que diseñar un plan estratégico ayudara a fortalecer la misión de la organización, y un 13\% no está de acuerdo. En relación al ítem 3, se tiene que el 93\% de la muestra cree que establecer una visión clara sobre el plan estratégico de la empresa permitirá a la organización alcanzar el éxito.

Estos resultados muestran como una visión clara tanto para la empresa como para los empleados puede cambiar la percepción de la misma, logrando así el éxito no solo de su funcionamiento sino también en su apertura al mercado. Por otra parte estos resultados, coinciden con lo encontrado por Deboya y Domínguez (2013) quienes determinaron que una visión clara en cualquier organización del ramo de prestación de servicio, puede permitirle su posicionamiento a la vanguardia del mercado. 


\section{Cuadro 3}

Indicador misión

ÍTEMS

$\mathrm{Si}$

NO

\begin{tabular}{|c|c|c|c|c|}
\hline & $\mathrm{F}$ & $\%$ & $\mathrm{~F}$ & $\%$ \\
\hline 7. ¿La empresa cuenta con una misión? & 3 & $20 \%$ & 12 & $80 \%$ \\
\hline $\begin{array}{l}\text { 8. ¿La empresa prestará un servicio de } \\
\text { acuerdo a su misión? }\end{array}$ & 2 & $13 \%$ & 13 & $87 \%$ \\
\hline $\begin{array}{l}\text { 9. ¿La misión enfoca los propósitos de } \\
\text { la organización? }\end{array}$ & 2 & $13 \%$ & 13 & $87 \%$ \\
\hline
\end{tabular}

El $80 \%$ de la muestra afirma que la empresa no cuenta con una misión clara y por lo tanto solo un 13\% afirma que la empresa prestaría sus servicios de acuerdo a la misión. Asimismo, solo el $13 \%$ cree que la misión se enfoca en los propósitos de la organización mientras que un $87 \%$ afirma que no. Estos resultados muestran el grado de desconocimiento de la mayoría de los empleados con respecto a la importancia de tener una misión clara en la empresa, por lo que se hace necesaria la intervención a través de este estudio.

Por otra parte, estos resultados confirman lo planteado por Koontz \& Weihrich (2011) quienes afirman que la aplicación principal de la misión es servir como una guía interna para quienes toman las decisiones importantes, y para que todos los proyectos y actividades puedan ser puestos a prueba en su compatibilidad con la misma. Es por ello que es necesario que la organización tenga claro cuál es su misión, y que todos los planes estratégicos deben ser cimentados en ella. 


\section{Cuadro 4}

Indicador políticas

$\begin{array}{lll}\text { ÍTEMS } & \mathrm{Si} & \text { NO }\end{array}$

\begin{tabular}{lllll} 
& $\mathrm{F}$ & $\%$ & $\mathrm{~F}$ & $\%$ \\
\hline $\begin{array}{l}\text { 10. ¿Existen políticas en la } \\
\text { empresa? }\end{array}$ & 5 & $33 \%$ & 10 & $67 \%$ \\
$\begin{array}{l}\text { 11. ¿Conoce Ud. las } \\
\text { políticas de la empresa? }\end{array}$ & 5 & $33 \%$ & 10 & $67 \%$ \\
$\begin{array}{l}\text { 12. ¿Es necesario } \\
\text { implementar nuevas } \\
\text { políticas en la empresa? }\end{array}$ & & & & \\
\hline
\end{tabular}

El 33\% de la muestra afirma que si existen políticas en la empresa mientras que el $67 \%$ cree que no. En este sentido, solo el $33 \%$ de la muestra la conoce como se observa en los resultados obtenidos para el ítem 11 y un $67 \%$ afirma que no las conoce. Finalmente, con respecto al ítem 12, el $87 \%$ concuerda que es necesario implementar nuevas políticas en la empresa, las cuales sean conocidas por todos.

Las políticas dentro de una empresa son de suma importancia ya que de acuerdo a lo planteado por Bauer (2014) las políticas implementadas en cualquier organización son principios generales que guían o canalizan el pensamiento y la acción en la toma de decisiones. Una política es una conducta que marca la compañía, y que sirve para describir su actitud, continuada en el tiempo, a la hora de enfrentarse a situaciones de diversa índole, desde situaciones laborales hasta administrativas y económicas. 


\section{Cuadro 5}

Indicador procedimientos

$\begin{array}{lll}\text { ÍTEMS } & \mathrm{Si} & \mathrm{NO}\end{array}$

\begin{tabular}{|c|c|c|c|c|}
\hline & $\mathrm{F}$ & $\%$ & $\mathrm{~F}$ & $\%$ \\
\hline $\begin{array}{l}\text { 13. ¿Sabe Ud. Cuáles son los } \\
\text { procedimientos que se llevan a } \\
\text { cabo en la empresa? }\end{array}$ & 4 & $27 \%$ & 11 & $73 \%$ \\
\hline $\begin{array}{l}\text { 14. ¿Está de acuerdo con esos } \\
\text { procedimientos? }\end{array}$ & 3 & $20 \%$ & 12 & $80 \%$ \\
\hline $\begin{array}{l}\text { 15. ¿Le gustaría que se crearan } \\
\text { nuevos procedimientos en la } \\
\text { empresa? }\end{array}$ & 13 & $87 \%$ & 2 & $13 \%$ \\
\hline
\end{tabular}

El 27\% de la muestra conoce los procedimientos que se desarrollan en la empresa mientras que un $73 \%$ no los conoce. En este mismo orden de ideas, el 20\% de la muestra está de acuerdo con los procedimientos existentes, mientras que un $80 \%$ no lo están. Finalmente al $87 \%$ de la muestra le gustaría que se crearan nuevos procedimientos y a un $20 \%$ no.

Estos resultados confirman la disposición de cambio y transformación evidenciados en la muestra estudiada, dado el alto porcentaje de la misma que insiste en la creación de nuevos procedimientos que sean conocidos y manejados por todos. Asimismo es importante acotar que estos resultados confirman lo afirmado por Fred (2013) quien establece que el desarrollo de una planificación estratégica con procedimientos claros y conocidos por todos los integrantes de la organización produce beneficios relacionados con la capacidad de realizar una gestión más eficiente, optimizando el uso recursos humanos y materiales, lo que redunda en la eficiencia productiva y en una mejor calidad de trabajo para todos los miembros 


\section{Cuadro 6}

Indicador programa

\begin{tabular}{|c|c|c|c|c|}
\hline \multirow{2}{*}{ ÍTEMS } & & \multicolumn{3}{|c|}{ NO } \\
\hline & $\mathrm{F}$ & $\%$ & $\mathrm{~F}$ & $\%$ \\
\hline $\begin{array}{l}\text { 16. ¿Sabe cuáles son los programas } \\
\text { por los que se rige la empresa? }\end{array}$ & 3 & $20 \%$ & 12 & $80 \%$ \\
\hline $\begin{array}{l}\text { 17. ¿Los programas le ofrecen } \\
\text { alternativas a la empresa? }\end{array}$ & 4 & $27 \%$ & 11 & $73 \%$ \\
\hline $\begin{array}{l}\text { ¿Proporcionan nuevas } \\
\text { alternativas? }\end{array}$ & 5 & $33 \%$ & 10 & $67 \%$ \\
\hline
\end{tabular}

El $20 \%$ de la muestra conoce los programas que rigen el funcionamiento de la empresa mientras que un $80 \%$ no los conoce. Asimismo, solo un $27 \%$ considera que los programas le ofrecen alternativas a la empresa mientras que un $73 \%$ no lo cree así. Por otra parte, solo un 33\% afirman que los programas pueden ofrecer nuevas alternativas mientras que un $67 \%$ no.

Estos resultados evidencian no solo el desconocimiento que posee la muestra sobre los programas que se utilizan en la empresa sino también su funcionalidad interna. Es necesario entonces que se establezcan nuevos lineamientos y programas que sean conocidos por todos y que a su vez sean integrados al funcionamiento de la organización. Estos resultados confirman lo planteado por Koontz \& Weihrich (2011), quienes afirman que los programas ayudan a revisar el carácter de curso de una acción requerida, entre varias alternativas y si los mismos no son manejados adecuadamente pueden incidir negativamente en el funcionamiento de la empresa. 


\section{Conclusiones}

En función de los resultados analizados, se concluye que es necesario el diseño de un plan estratégico para la empresa objeto de estudio, por cuanto los principios de la planificación estratégica le permitirá a los trabajadores estar capacitados para resolver cualquier eventualidad y potenciar así el crecimiento de la empresa, siendo pertinente que estén formados para la aplicación del plan estratégico, situación que les permitirá contar con mayores posibilidades de no sucumbir ante el mercado voraz, el cual cada día se hace riguroso con las empresas que no se fundamentan desde los principios científicos de la gerencia, así como no asumen acciones para dominar el mercado.

\section{Referencias}

1. Allen, C. (2010). Sentido de Urgencia. Recuperado de www.amte.org.mx/portal/wpcontent/.../2010/11/El_ciclo_del_exito.pdf

2. Bauer, S. (2014). Estructura del Plan Estratégico. Panapo editores.

3. Chiavenato (2006) comprende su cultura. Publicado en: virtual.urbe.edu/tesispub/0094116/cap02.

4. Chiavenato (2006) asumir los cambio en la cultura empresarial. Publicado en: http://virtual.urbe.edu/tesispub/0094116/cap02.pdf

5. Collins, J. y Porras, J. (1995). Empresas que perduran. Norma, Bogotá.

6. Deboya y Domínguez (2013) Propuesta de un plan estratégico para la empresa FUNDELEC LTDA, para afrontar el impacto del Tratado de Libre Comercio entre Colombia y Estados Unidos. Recuperado de http://red.uao.edu.co/handle/10614/4822

7. Fred, D. (2003) planeación estratégicas, etapas. Recuperado de http://thesmadruga2.blogspot.com/2013/05/planeacion-estrategica.html.

8. Hernández, R., Fernández, C., Baptista, P. (2014). Metodología de la Investigación. México: Editorial Mc - Graw - Hill Interamericana. 
9. Koontz, H y Weihrich, H (2007) "Elementos de Administración, un Enfoque Internacional". McGraw-Hill Interamericana Editores, S.A. de C.V. México.

10. Kotler, P. \& Lee, N. (2011), Marketing en el sector público, México: Fondo de cultura económica.

11. Kenichi (1991) estrategias que ponen de relieve sus valores y juegan con sus fortalezas organizativas

12. Ned (2014) objetivos y metas de un plan estratégico. Recuperado de http://www.aiteco.com/planes-estrategicos.20

13. Sainz de Vicuña y Milla Gutiérrez (2012). Plan y estrategia. Recuperado de https://prezi.com/1wyzsqbfmifx/planeacion-estrategica/

\section{References}

1. Allen, C. (2010). Sense of urgency. Recovered from www.amte.org.mx/portal/wpcontent/.../2010/11/El_ciclo_del_exito.pdf

2. Bauer, S. (2014). Structure of the Strategic Plan. Panapo editors.

3. Chiavenato (2006) understands its culture. Published at: virtual.urbe.edu/tesispub/0094116/cap02.

4. Chiavenato (2006) assume the changes in business culture. Published at: http://virtual.urbe.edu/tesispub/0094116/cap02.pdf

5. Collins, J. and Porras, J. (1995). Companies that last. Norma, Bogotá.

6. Deboya and Domínguez (2013) Proposal of a strategic plan for the company FUNDELEC LTDA, to face the impact of the Free Trade Agreement between Colombia and the United States. Recovered from http://red.uao.edu.co/handle/10614/4822

7. Fred, D. (2003) strategic planning, stages. Recovered from http://thesmadruga2.blogspot.com/2013/05/planeacion-estrategica.html. 


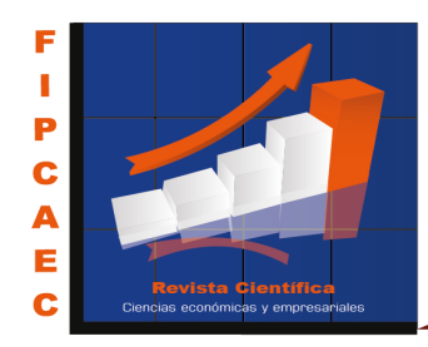

8. Hernández, R., Fernández, C., Baptista, P. (2014). Investigation methodology. Mexico: Editorial Mc - Graw - Interamerican Hill.

9. Koontz, $\mathrm{H}$ and Weihrich, $\mathrm{H}$ (2007) "Elements of Administration, an International Approach". McGraw-Hill Interamericana Editores, S.A. from C.V. Mexico.

10. Kotler, P. \& Lee, N. (2011), Marketing in the public sector, Mexico: Economic culture fund.

11. Kenichi (1991) strategies that highlight their values and play with their organizational strengths

12. Ned (2014) objectives and goals of a strategic plan. Recovered from http://www.aiteco.com/planes-estrategicos.20

13. Sainz de Vicuña and Milla Gutiérrez (2012). Plan and strategy Recovered from https://prezi.com/1wyzsqbfmifx/planeacion-estrategica/ 